\title{
DIRICHLET PROBLEMS WITH SINGULAR AND GRADIENT QUADRATIC LOWER ORDER TERMS
}

\author{
LUCIO BOCCARDO $^{1}$
}

\begin{abstract}
We present a revisited form of a result proved in [Boccardo, Murat and Puel, Portugaliae Math. 41 (1982) 507-534] and then we adapt the new proof in order to show the existence for solutions of quasilinear elliptic problems also if the lower order term has quadratic dependence on the gradient and singular dependence on the solution.
\end{abstract}

Mathematics Subject Classification. 35J20, 35J25, 35J65.

Received January 8, 2008.

Published online April 26, 2008.

Dédié à Jean-Pierre Puel pour ses 60 ans avec $3^{3}$ ans $\mid D$ amitié $\left.\right|^{2}$

\section{INTRODUCTION}

Quasilinear Dirichlet problems having lower order terms with superlinear growth with respect to the gradient play a fundamental role in the study of Nonlinear Differential Equations.

We recall the paper [30], by Jean-Pierre Puel, for its influence on later developments.

Moreover, quasilinear Dirichlet problems having lower order terms with quadratic growth with respect to the gradient arise naturally in Calculus of Variations and in Stochastic Control.

For example, if we consider the functional (in all the paper $\Omega$ is a bounded open set in $\mathbb{R}^{N}$ )

$$
J(v)=\frac{1}{2} \int_{\Omega}\left(1+|v|^{r}\right)|D v|^{2}-\int_{\Omega} f(x) v(x), \quad r>1,
$$

the Euler-Lagrange equation is

$$
u \in W_{0}^{1,2}(\Omega):-\operatorname{div}\left(\left(1+|u|^{r}\right) D u\right)+\frac{r}{2}|u|^{r-2} u|D u|^{2}=f
$$

The direct study of Dirichlet problems similar to the previous ones gives some difficulties. The first difficulty is due to the fact that the principal part of the differential operator $-\operatorname{div}\left(\left(1+|v|^{r}\right) D v\right)$ is not well defined on the whole $W_{0}^{1,2}(\Omega)$. The second and main one is that the lower order term $|v|^{r-2} v|D v|^{2}$ not only is not well

Keywords and phrases. Quadratic gradient, singular lower order term.

1 Dipartimento di Matematica, Università di Roma 1, Piazza A. Moro 2, 00185 Roma, Italy; boccardo@mat.uniroma1.it 
defined on the whole $W_{0}^{1,2}(\Omega)$, but, even if $v \in L^{\infty}(\Omega) \cap W_{0}^{1,2}(\Omega),|v|^{r-2} v|D v|^{2}$ does not belong to $W^{-1,2}(\Omega)$. However, the lower order term has the useful property that $v \cdot\left(|v|^{r-2} v|D v|^{2}\right) \geq 0$.

In a more general setting, in Section 2, we present a revisited form of the techniques of [13] (and of [9]) in order to study the Dirichlet problem

$$
u \in W_{0}^{1,2}(\Omega):-\operatorname{div}(M(x, u) D u)+g(x, u, D u)=f
$$

where on the right hand side $f$ we assume that

$$
f \in L^{m}(\Omega), 1 \leq m \leq N / 2
$$

and whose simplest example is

$$
u \in W_{0}^{1,2}(\Omega):-\Delta u+u|D u|^{2}=f .
$$

Our assumptions follow from the model problem (1.2). We assume that $M(x, s): \Omega \times \mathbb{R} \rightarrow \mathbb{R}^{N^{2}}, g(x, s, \xi)$ : $\Omega \times \mathbb{R} \times \mathbb{R}^{N} \rightarrow \mathbb{R}$ are functions which are measurable with respect to $x$ and continuous with respect to $s$ and $\xi$, such that, for $x \in \Omega, s \in \mathbb{R}, \xi \in \mathbb{R}^{N}$, we have

$$
\begin{gathered}
\alpha|\xi|^{2} \leq M(x, s) \xi \xi, \quad|M(x, s)| \leq \beta(s), \\
|g(x, s, \xi)| \leq \gamma(s)|\xi|^{2}, \\
g(x, s, \xi) s \geq \nu(|s|)|s||\xi|^{2},
\end{gathered}
$$

where $\alpha>0, \beta(s), \gamma(s)$ are continuous, increasing (possibly unbounded) functions of a real variable and $\nu(s)$ : $\mathbb{R}^{+} \rightarrow \mathbb{R}^{+}$is continuous, increasing and $\nu(0)=0$.

Recall that in order to study (1.3), if the right hand side belongs to $L^{2}(\Omega)$, it is enough the slightly weaker assumption $g(x, s, \xi) s \geq 0$, introduced in [13].

Thanks to the presence of the lower order term with quadratic dependence with respect to the gradient and to the assumption (1.7), introduced in [9], the Dirichlet problem (1.3) is allowed to have finite energy weak solutions (see [9]), even if $f$ belongs only to $L^{1}(\Omega)$. This result (regularizing effect of $g$ ) is somewhat surprising because it is not true in the linear case (for example if $g(x, s, \xi) \equiv 0$ ).

Contributions to the existence of solutions of nonlinear elliptic problems with lower order terms having quadratic growth with respect to the gradient, like (1.3), can be found in some papers in collaboration with F. Murat and J.-P. Puel [14-16] (see also [7,19,20]), where we proved existence of bounded solutions (without the assumption $g(x, s, \xi) s \geq 0$ and with the assumption $\left.f \in L^{m}(\Omega), m>N / 2\right)$.

If we look for unbounded solutions, we refer to the paper [13], in collaboration with F. Murat and J.-P. Puel and to

- [4,21]: unbounded solutions and data in the dual space (with the assumption $g(x, s, \xi) s \geq 0$ );

- $[9,10,17,27,29]:$ unbounded solutions and $f \in L^{1}(\Omega)$ (with the assumption (1.7));

- [6], where a different notion of solution is used (with the assumption (1.7)).

Remark 1.1. Thanks to the assumption $g(x, s, \xi) s \geq 0$ and to Stampacchia type $L^{\infty}$-estimates [31], the existence results for the case $m>N / 2$ are contained in [16].

In the last two sections, we adapt the techniques of the second section in order to prove the existence of strictly positive (in $\Omega$ ) solutions of two Dirichlet problems with lower order term having quadratic dependence on the gradient and singular dependence on $u$. 
Simple examples of the existence results of the last two sections are

$$
\begin{gathered}
u \in W_{0}^{1,2}(\Omega), u>0 \text { in } \Omega:-\alpha \Delta u+\frac{|D u|^{2}}{u^{\theta}}=f, \quad \alpha>0,0<\theta<1 . \\
u \in W_{0}^{1,2}(\Omega), u>0 \text { in } \Omega:-\alpha \Delta u+\frac{|D u|^{2}}{u}=f, \quad \alpha>1 .
\end{gathered}
$$

Our results are closely related to those of [1-3,22].

For the sake of simplicity, we confine our study to the framework: positive data, right hand side functions (instead of measures absolutely continuous with respect to the capacity, as in [10]), $W_{0}^{1,2}(\Omega)$-solutions instead of $W_{0}^{1, p}(\Omega)$-solutions and linear dependence with respect to the gradient in the principal part of the differential operator.

\section{The BMP-Portugaliae Mathematica Method REvisited}

In this section, we shall follow the approach of the paper [13] (in collaboration with F. Murat and J.-P. Puel and published in Portugaliae Mathematica), proving a more general result, thanks to some new techniques (mainly due to [9]).

Theorem 2.1. Assume (1.4), (1.5), (1.6), (1.7) and $\beta(s) \leq \beta_{0}|s|^{p}$, where $p<m^{* *}$. Then there exists $u \in$ $W_{0}^{1,2}(\Omega)$ with $|M(x, u) D u| \in L^{r}(\Omega), r=\frac{2 m^{* *}}{p+m^{* *}}$, and $g(x, u, D u) \in L^{1}(\Omega)$, such that

$$
\int_{\Omega} M(x, u) D u D \phi+\int_{\Omega} g(x, u, D u) \phi=\int_{\Omega} f \phi, \quad \forall \phi \in \mathcal{D}(\Omega) .
$$

Before the proof of the theorem, we shall prove some preliminary results.

Let the truncation $T_{k}: \mathbb{R} \mapsto \mathbb{R}$ be defined by

$$
T_{k}(t)=\left\{\begin{array}{cl}
t, & \text { if }|t| \leq k \\
k \frac{t}{|t|}, & \text { if }|t|>k
\end{array}\right.
$$

and let $f_{n}(x)=T_{n}[f(x)]$, so that $f_{n} \in L^{\infty}(\Omega),\left|f_{n}\right| \leq|f|$ and $\left\|f_{n}-f\right\|_{L^{1}(\Omega)} \rightarrow 0$. Consider the boundary value problems

$$
\left\{\begin{array}{c}
-\operatorname{div}\left(M\left(x, u_{n}\right) D u_{n}\right)+g\left(x, u_{n}, D u_{n}\right)+\frac{1}{n} u_{n}=f_{n}, \text { in } \Omega ; \\
u_{n}=0, \text { on } \partial \Omega .
\end{array}\right.
$$

Since $f_{n}$ is a bounded function, by a result of [16], there exists $u_{n} \in W_{0}^{1,2}(\Omega) \cap L^{\infty}(\Omega)$ weak solution of (2.2):

$$
\int_{\Omega} M\left(x, u_{n}\right) D u_{n} D \phi+\int_{\Omega} g\left(x, u_{n}, D u_{n}\right) \phi+\frac{1}{n} \int_{\Omega} u_{n} \phi=\int_{\Omega} f_{n} \phi,
$$

$\forall \phi \in W_{0}^{1,2}(\Omega) \cap L^{\infty}(\Omega)$.

Lemma 2.1. If $f \in L^{1}(\Omega)$, then there exists $R>0$ (see [9]) such that

$$
\left\|u_{n}\right\|_{W_{0}^{1,2}(\Omega)} \leq R
$$


Proof. The use of $T_{j}\left(u_{n}\right)$ as test function in (2.2) implies

$$
\begin{gathered}
\int_{\Omega} M\left(x, u_{n}\right) D u_{n} D T_{j}\left(u_{n}\right)+\int_{\Omega} g\left(x, u_{n}, D u_{n}\right) T_{j}\left(u_{n}\right) \leq \int_{\Omega} f_{n} T_{j}\left(u_{n}\right) \\
\alpha \int_{\Omega}\left|D T_{j}\left(u_{n}\right)\right|^{2}+j \int_{\left\{j<\left|u_{n}\right|\right\}} \nu\left(\left|u_{n}\right|\right)\left|D u_{n}\right|^{2} \leq j \int_{\Omega}|f| \\
\alpha \int_{\left\{\left|u_{n}\right| \leq j\right\}}\left|D u_{n}\right|^{2}+j \nu(j) \int_{\left\{j<\left|u_{n}\right|\right\}}\left|D u_{n}\right|^{2} \leq j \int_{\Omega}|f| .
\end{gathered}
$$

Then it follows

$$
\int_{\Omega}\left|D u_{n}\right|^{2}=\int_{\left\{\left|u_{n}\right| \leq j\right\}}\left|D u_{n}\right|^{2}+\int_{\left\{j<\left|u_{n}\right|\right\}}\left|D u_{n}\right|^{2} \leq\left[\frac{j}{\alpha}+\frac{1}{\nu(j)}\right] \int_{\Omega}|f| .
$$

Thus the sequence $\left\{u_{n}\right\}$ is bounded in $W_{0}^{1,2}(\Omega)$ : we can say that (up to a subsequence still denoted by $\left\{u_{n}\right\}$ ) the sequence converges weakly in $W_{0}^{1,2}(\Omega)$ and a.e. to $u$, for some $u \in W_{0}^{1,2}(\Omega)$.

Lemma 2.2. The following inequality holds (see [9])

$$
\int_{\left\{k \leq\left|u_{n}\right|\right\}}\left|g\left(x, u_{n}, D u_{n}\right)\right| \leq \int_{\left\{k \leq\left|u_{n}\right|\right\}}|f|, \quad \forall k \geq 0 .
$$

Proof. Let $\epsilon>0$ and $k \geq 0$. The use of $T_{1}\left\{\frac{1}{\epsilon}\left[u_{n}-T_{k}\left(u_{n}\right)\right]\right\}$ as test function in (2.2) gives

$$
\int_{\Omega} g\left(x, u_{n}, D u_{n}\right) T_{1}\left\{\frac{1}{\epsilon}\left[u_{n}-T_{k}\left(u_{n}\right)\right]\right\} \leq \int_{\Omega}|f| .
$$

Then letting $\epsilon \rightarrow 0(k \geq 0)$ the previous estimate implies (2.3).

Lemma 2.3. The sequence $\left\{D u_{n}(x)\right\}$ converges a.e. to $D u(x)$.

Proof. The test function used in this proof is the same used in [5] (for similar results see also $[8,12]$ ). Thanks to (2.3), we have from (2.2) with test function $T_{h}\left[u_{n}-T_{k}(u)\right]$,

$$
\int_{\Omega} M\left(x, u_{n}\right) D u_{n} D T_{h}\left[u_{n}-T_{k}(u)\right]+\frac{1}{n} \int_{\Omega} u_{n} T_{h}\left[u_{n}-T_{k}(u)\right] \leq 2 \int_{\Omega}|f|\left|T_{h}\left[u_{n}-T_{k}(u)\right]\right|
$$

which gives

$$
\mid \begin{aligned}
& \int_{\Omega}\left[M\left(x, u_{n}\right) D u_{n}-M\left(x, u_{n}\right) D T_{k}(u)\right] D T_{h}\left[u_{n}-T_{k}(u)\right]+\frac{1}{n} \int_{\Omega} u_{n} T_{h}\left[u_{n}-T_{k}(u)\right] \\
& \leq 2 h \int_{\Omega}|f|-\int_{\Omega} M\left(x, u_{n}\right) D T_{k}(u) D T_{h}\left[u_{n}-T_{k}(u)\right] .
\end{aligned}
$$

Thus it follows

$$
\alpha \limsup _{n \rightarrow \infty} \int_{\Omega}\left|D T_{h}\left[u_{n}-T_{k}(u)\right]\right|^{2} \leq 2 h \int_{\Omega}|f|
$$


Let now $q$ be such that $1<q<2$. Then we have

$$
\mid \begin{aligned}
& \int_{\Omega}\left|D\left(u_{n}-u\right)\right|^{q}=\int_{\left\{\left|u_{n}-u\right| \leq h,|u| \leq k\right\}}\left|D\left(u_{n}-u\right)\right|^{q} \\
& +\int_{\left\{\left|u_{n}-u\right| \leq h,|u|>k\right\}}\left|D\left(u_{n}-u\right)\right|^{q}+\int_{\left\{\left|u_{n}-u\right|>h\right\}}\left|D\left(u_{n}-u\right)\right|^{q} \\
& \leq \int_{\Omega}\left|D T_{h}\left[u_{n}-T_{k}(u)\right]\right|^{q} \\
& +2^{q-1} R^{q} \operatorname{meas}\{|u|>k\}^{1-\frac{q}{2}}+2^{q-1} R^{q} \operatorname{meas}\left\{\left|u_{n}-u\right|>h\right\}^{1-\frac{q}{2}} .
\end{aligned}
$$

Thus, for every $h>0$,

$$
\limsup _{n \rightarrow \infty} \int_{\Omega}\left|D\left(u_{n}-u\right)\right|^{q} \leq\left[h \frac{2}{\alpha} \int_{\Omega}|f|\right]^{\frac{q}{2}}|\Omega|^{1-\frac{q}{2}}+2^{q-1} R^{q} \operatorname{meas}\{|u|>k\}^{1-\frac{q}{2}}
$$

That is, letting $h \rightarrow 0$ and then $k \rightarrow+\infty$,

$$
\int_{\Omega}\left|D u_{n}-D u\right|^{q} \rightarrow 0, \quad \forall q<2
$$

Then (up to subsequences) $D u_{n}(x)$ converges a.e. to $D u(x)$.

Fatou Lemma and (2.3), written for $k=0$, imply the following inequality.

\section{Corollary 2.1.}

$$
\int_{\Omega}|g(x, u, D u)| \leq \int_{\Omega}|f|
$$

In the following lemma, a summability result is proved, in the spirit of $[11,31]$; but, thanks to the presence of the lower order term, it is possible to prove extra summability, as in [25].

Lemma 2.4. If $f \in L^{m}(\Omega), 1<m<\frac{N}{2}$, the sequence $\left\{u_{n}\right\}$ is bounded in $L^{2 m^{* *}}(\Omega)$, where $m^{* *}=\left(m^{*}\right)^{*}=$ $\frac{m N}{N-2 m}$.

Proof. Let $\gamma=\frac{N(m-1)}{N-2 m}$, so that $(\gamma+1) 2^{*}=2 \gamma m^{\prime}=2 m^{* *}$. The use of $\left|u_{n}\right|^{2 \gamma-1} u_{n}$ as test function in (2.2) yields, for fixed $j$,

$$
\int_{\left\{j<\left|u_{n}\right|\right\}} g\left(x, u_{n}, D u_{n}\right)\left|u_{n}\right|^{2 \gamma-1} u_{n} \leq \int_{\Omega}|f|\left|u_{n}\right|^{2 \gamma}
$$

which implies

$$
\nu(j) \int_{\left\{j<\left|u_{n}\right|\right\}}\left|u_{n}\right|^{2 \gamma}\left|D u_{n}\right|^{2} \leq\|f\|_{L^{m}(\Omega)}\left[\int_{\Omega}\left|u_{n}\right|^{(2 \gamma-2) m^{\prime}}\right]^{\frac{1}{m^{\prime}}}
$$


Then

$$
\mid \begin{aligned}
& \frac{S^{2}}{(\gamma+1)^{2}}\left[\int_{\Omega}\left|u_{n}\right|^{(\gamma+1) 2^{*}}\right]^{\frac{2}{2^{*}}} \leq \int_{\Omega}\left|u_{n}\right|^{2 \gamma}\left|D u_{n}\right|^{2} \\
& \leq j^{2 \gamma} \int_{\left\{\left|u_{n}\right| \leq j\right\}}\left|D u_{n}\right|^{2}+\frac{1}{\nu(j)}\|f\|_{L^{m}(\Omega)}\left[\int_{\Omega}\left|u_{n}\right|^{2 \gamma m^{\prime}}\right]^{\frac{1}{m^{\prime}}} \\
& \leq j^{2 \gamma} R^{2}+\frac{1}{\nu(j)}\|f\|_{L^{m}(\Omega)}\left[\int_{\Omega}\left|u_{n}\right|^{(\gamma+1) 2^{*}}\right]^{\frac{1}{m^{\prime}}}
\end{aligned}
$$

which implies that the sequence $\left\{u_{n}\right\}$ is bounded in $L^{2 m^{* *}}(\Omega)$.

Remark 2.1. Note that Lemmas 2.1 and 2.4 cover all the interval $1 \leq m<N / 2$.

Lemma 2.5. Under the assumptions of Theorem 2.1, the sequence $\left\{M\left(x, u_{n}\right) D u_{n}\right\}$ converges to $M(x, u) D u$ weakly in $L^{r}(\Omega), r=\frac{2 m^{* *}}{p+m^{* *}}$.

Proof. Fix $r>1$ such that $\frac{2 p r}{2-r}<2 m^{* *}$. The inequality

$$
\int_{\Omega}\left|M\left(x, u_{n}\right) D u_{n}\right|^{r} \leq \beta_{0}^{r} \int_{\Omega}\left|u_{n}\right|^{p r}\left|D u_{n}\right|^{r} \leq \beta_{0}^{r}\left[\int_{\Omega}\left|u_{n}\right|^{\frac{2 p r}{2-r}}\right]^{\frac{2-r}{2}}\left[\int_{\Omega}\left|D u_{n}\right|^{2}\right]^{\frac{r}{2}}
$$

implies that the sequence $\left\{M\left(x, u_{n}\right) D u_{n}\right\}$ is bounded in $L^{r}(\Omega)$, since $\frac{2 p r}{2-r}=2 m^{* *}$. Then the sequence $\left\{M\left(x, u_{n}\right) D u_{n}\right\}$ converges strongly in $L^{s}(\Omega), s>1$, to $M(x, u) D u$, for every $1<s<r$ and weakly in $L^{r}(\Omega)$.

Proof of Theorem 2.1. For the sake of simplicity, we present the proof in the easy case $f(x) \geq 0$, which implies $u_{n}(x) \geq 0$ and, thanks to the assumption (1.7), $g\left(x, u_{n}, D u_{n}\right) \geq 0$.

We point out that the test functions used in this proof are similar to those used in [13]. The proof proceeds by steps.

First step. By (2.2) we have

$$
\int_{\Omega} M\left(x, u_{n}\right) D u_{n} D \phi+\int_{\Omega} g\left(x, u_{n}, D u_{n}\right) \phi \leq \int_{\Omega} f_{n} \phi,
$$

for every $0 \leq \phi \in \mathcal{D}(\Omega)$. Since $M\left(x, u_{n}\right) D u_{n}$ converges weakly in $L^{r}$ to $M(x, u) D u$ and $g\left(x, u_{n}, D u_{n}\right) \geq 0$ converges a.e. to $g(x, u, D u) \geq 0$, Fatou Lemma yields

$$
\int_{\Omega} M(x, u) D u D \phi+\int_{\Omega} g(x, u, D u) \phi \leq \int_{\Omega} f \phi .
$$


Second step. As before $0 \leq \phi \in \mathcal{D}(\Omega)$. Define $H(t)=\int_{0}^{t} \gamma(s) \mathrm{d} s$ and use $\mathrm{e}^{-\frac{1}{\alpha} H\left(u_{n}\right)} \mathrm{e}^{\frac{1}{\alpha} H\left(T_{k}(u)\right)} \phi$ as test function. We obtain

$$
\mid \begin{aligned}
& \int_{\Omega} M\left(x, u_{n}\right) D u_{n} D \phi \mathrm{e}^{-\frac{1}{\alpha} H\left(u_{n}\right)} \mathrm{e}^{\frac{1}{\alpha} H\left(T_{k}(u)\right)} \\
& +\frac{1}{\alpha} \int_{\Omega} M\left(x, u_{n}\right) D u_{n} D T_{k}(u) \gamma\left(T_{k}(u)\right) \mathrm{e}^{-\frac{1}{\alpha} H\left(u_{n}\right)} \mathrm{e}^{\frac{1}{\alpha} H\left(T_{k}(u)\right)} \phi \\
& +\frac{1}{n} \int_{\Omega} u_{n} \phi=\int_{\Omega} f_{n} \mathrm{e}^{-\frac{1}{\alpha} H\left(u_{n}\right)} \mathrm{e}^{\frac{1}{\alpha} H\left(T_{k}(u)\right)} \phi \\
& +\frac{1}{\alpha} \int_{\Omega} M\left(x, u_{n}\right) D u_{n} D u_{n} \gamma\left(u_{n}\right) \mathrm{e}^{-\frac{1}{\alpha} H\left(u_{n}\right)} \mathrm{e}^{\frac{1}{\alpha} H\left(T_{k}(u)\right)} \phi \\
& -\int_{\Omega} g\left(x, u_{n}, D u_{n}\right) \mathrm{e}^{-\frac{1}{\alpha} H\left(u_{n}\right)} \mathrm{e}^{\frac{1}{\alpha} H\left(T_{k}(u)\right)} \phi \geq 0 .
\end{aligned}
$$

The limit $n \rightarrow \infty$, Fatou Lemma and Lemma 2.5 yield

$$
\mid \begin{aligned}
& \int_{\Omega} M(x, u) D u D \phi \mathrm{e}^{-\frac{1}{\alpha} H(u)} \mathrm{e}^{\frac{1}{\alpha} H\left(T_{k}(u)\right)} \\
& +\frac{1}{\alpha} \int_{\Omega} M(x, u) D u D T_{k}(u) \gamma\left(T_{k}(u)\right) \mathrm{e}^{-\frac{1}{\alpha} H(u)} \mathrm{e}^{\frac{1}{\alpha} H\left(T_{k}(u)\right)} \phi \\
& \geq \int_{\Omega} f \mathrm{e}^{-\frac{1}{\alpha} H(u)} \mathrm{e}^{\frac{1}{\alpha} H\left(T_{k}(u)\right)} \phi \\
& \frac{1}{\alpha} \int_{\Omega} M(x, u) D u D u \gamma(u) \mathrm{e}^{-\frac{1}{\alpha} H(u)} \mathrm{e}^{\frac{1}{\alpha} H\left(T_{k}(u)\right)} \phi-\int_{\Omega} g(x, u, D u) \mathrm{e}^{-\frac{1}{\alpha} H(u)} \mathrm{e}^{\frac{1}{\alpha} H\left(T_{k}(u)\right)} \phi
\end{aligned}
$$

which implies

$$
\mid \begin{aligned}
& \int_{\Omega} M(x, u) D u D \phi \mathrm{e}^{-\frac{1}{\alpha} H(u)} \mathrm{e}^{\frac{1}{\alpha} H\left(T_{k}(u)\right)} \geq \int_{\Omega} f \mathrm{e}^{-\frac{1}{\alpha} H(u)} \mathrm{e}^{\frac{1}{\alpha} H\left(T_{k}(u)\right)} \phi \\
& -\int_{\Omega} g(x, u, D u) \mathrm{e}^{-\frac{1}{\alpha} H(u)} \mathrm{e}^{\frac{1}{\alpha} H\left(T_{k}(u)\right)} \phi
\end{aligned}
$$

In order to use Lebesgue Theorem $($ as $k \rightarrow+\infty)$ in the previous inequality, note that $\frac{\mathrm{e}^{\frac{1}{\alpha} H\left(T_{k}(u)\right)}}{\mathrm{e}^{\frac{1}{\alpha} H(u)}} \leq 1$. Then

$$
\int_{\Omega} M(x, u) D u D \phi \geq \int_{\Omega} f \phi-\int_{\Omega} g(x, u, D u) \phi .
$$

The inequalities (2.4) and (2.5) implies

$$
\int_{\Omega} M(x, u) D u D \phi+\int_{\Omega} g(x, u, D u) \phi=\int_{\Omega} f \phi, \quad \forall 0 \leq \phi \in W_{0}^{1, \infty}(\Omega) .
$$

Since we can write $\varphi=\varphi^{+}+\varphi^{-}$for every $\varphi \in W_{0}^{1, \infty}(\Omega)$, we proved the existence of a solution $u$ of the Dirichlet problem (1.3). 


\section{A LOWER ORDER TERM SINGULAR With RESPECT TO $u$ (SUBLINEAR GROWTH SINGULARITY)}

Now we study the existence of weak solution for some elliptic problems with lower order terms having quadratic growth with respect to the gradient and singular dependence with respect to the solution.

A model problem is the Euler-Lagrange equation (1.2) if (at least formally) in (1.1) we assume $0<r<1$. Other motivations can be found in $[1,2,22]$.

Here we assume more summability on the right hand side:

$$
\begin{gathered}
0 \leq f \in L^{m}(\Omega), \quad m \geq\left(\frac{2^{*}}{\theta}\right)^{\prime}, \quad f \not \equiv 0, \\
0<\theta<1 .
\end{gathered}
$$

Moreover, let $Q(x, s): \Omega \times \mathbb{R} \rightarrow \mathbb{R}^{N^{2}}$ symmetric, measurable with respect to $x$ and continuous with respect to $s$ such that, for $x \in \Omega, s \in \mathbb{R}$ we have

$$
a|\xi|^{2} \leq Q(x, s) \xi \xi \leq b|\xi|^{2}, \quad 0<a \leq b
$$

Theorem 3.1. Under the assumptions (1.5), with $\beta(s) \leq \beta_{0} \in \mathbb{R}^{+},(3.1)$, (3.2) and (3.3), there exists $u \in$ $W_{0}^{1,2}(\Omega) \cap L^{\infty}(\Omega)$, verifying $u>0$ in $\Omega$, and $\frac{Q(x, u) D u D u}{u^{\theta}} \in L^{1}(\Omega)$, such that

$$
\int_{\Omega} M(x, u) D u D \phi+\int_{\Omega} \frac{Q(x, u) D u D u}{u^{\theta}} \phi=\int_{\Omega} f \phi, \quad \forall \phi \in W_{0}^{1,2}(\Omega) \cap L^{\infty}(\Omega) .
$$

Proof. Let $0<\varepsilon<1$ and consider $u_{\varepsilon} \in W_{0}^{1,2}(\Omega) \cap L^{\infty}(\Omega)$ :

$$
\left\{\begin{array}{c}
-\operatorname{div}\left(M\left(x, u_{\varepsilon}\right) D u_{\varepsilon}\right)+\frac{Q\left(x, u_{\varepsilon}\right) D u_{\varepsilon} D u_{\varepsilon}}{\left(\varepsilon+u_{\varepsilon}\right)^{\theta}}=f_{\varepsilon}, \text { in } \Omega \\
u_{\varepsilon}=0, \quad \text { on } \partial \Omega
\end{array}\right.
$$

where $\left\{f_{\varepsilon}\right\}$ is a sequence of bounded functions converging to $f$ in $L^{m}(\Omega), 0 \leq f_{\varepsilon} \leq f ;$ e.g. $f_{\varepsilon}=T_{\frac{1}{\varepsilon}}(f)$. Note that $u_{\varepsilon}$ exists by Theorem 2.1 and that $u_{\varepsilon} \geq 0$ by (3.1). Let $\delta>0$. We use $\left[\left(u_{\varepsilon}+\delta\right)^{\theta}-\delta^{\theta}\right]$ as test function and we have (once more, thanks to the use of the lower order term as leader term, as in [25])

$$
\int_{\Omega} \frac{Q\left(x, u_{\varepsilon}\right) D u_{\varepsilon} D u_{\varepsilon}}{\left(\varepsilon+u_{\varepsilon}\right)^{\theta}}\left[\left(u_{\varepsilon}+\delta\right)^{\theta}-\delta^{\theta}\right] \leq \int_{\Omega} f_{\varepsilon}\left[\left(u_{\varepsilon}+\delta\right)^{\theta}-\delta^{\theta}\right]
$$

The limit $\delta \rightarrow 0$ implies

$$
\frac{a}{(\varepsilon+1)^{\theta}} \int_{\left\{1<u_{\varepsilon}\right\}}\left|D u_{\varepsilon}\right|^{2} \leq a \int_{\left\{1<u_{\varepsilon}\right\}} \frac{\left|D u_{\varepsilon}\right|^{2} u_{\varepsilon}^{\theta}}{\left(\varepsilon+u_{\varepsilon}\right)^{\theta}} \leq \int_{\Omega} f_{\varepsilon} u_{\varepsilon}^{\theta} .
$$

Furthermore the use of $T_{1}\left(u_{\varepsilon}\right)$ as test function produces

$$
\alpha \int_{\Omega}\left|D T_{1}\left(u_{\varepsilon}\right)\right|^{2} \leq\left\|f_{\varepsilon}\right\|_{1}
$$

so that

$$
\int_{\Omega}\left|D u_{\varepsilon}\right|^{2} \leq \frac{\left\|f_{\varepsilon}\right\|_{1}}{\alpha}+\frac{2^{\theta}}{a} \int_{\Omega} f_{\varepsilon} u_{\varepsilon}^{\theta} .
$$


Then assumption (3.1) and Sobolev inequality imply that the sequence $\left\{u_{\varepsilon}\right\}$ is bounded in $W_{0}^{1,2}(\Omega)$ (thus $u_{\varepsilon} \rightarrow u$, for some $u \in W_{0}^{1,2}(\Omega)$ ). As in the previous section, it is possible to prove that the sequence of lower order terms is bounded in $L^{1}(\Omega)$, i.e.

$$
\int_{\Omega} \frac{Q\left(x, u_{\varepsilon}\right) D u_{\varepsilon} D u_{\varepsilon}}{\left(\varepsilon+u_{\varepsilon}\right)^{\theta}} \leq \int_{\Omega}\left|f_{\varepsilon}\right|
$$

and that $D u_{\varepsilon}(x)$ converges a.e. to $D u(x)$.

Define, for $t \geq 0$,

$$
H_{\varepsilon}(t)=\frac{(\varepsilon+t)^{1-\theta}}{(1-\theta)}, H_{\varepsilon}^{\prime}(t)=\frac{1}{(\varepsilon+t)^{\theta}} ; H_{0}(t)=\frac{t^{1-\theta}}{(1-\theta)}
$$

Now we shall prove that $u>0$ in $\Omega$. Indeed, take $\varphi=\mathrm{e}^{-b \frac{H_{\varepsilon}\left(u_{\varepsilon}\right)}{\alpha}} \phi$, with $\phi \in \mathcal{D}(\Omega), \phi \geq 0$, as test function in (3.4), using assumptions (1.5) and (3.3), we get

$$
\mid \begin{aligned}
& \int_{\Omega} M\left(x, u_{\varepsilon}\right) D u_{\varepsilon} D \phi \mathrm{e}^{-b \frac{H_{\varepsilon}\left(u_{\varepsilon}\right)}{\alpha}}-\int_{\Omega} f_{\varepsilon} \mathrm{e}^{-b \frac{H_{\varepsilon}\left(u_{\varepsilon}\right)}{\alpha} \phi} \\
& =\frac{b}{\alpha} \int_{\Omega} \frac{M\left(x, u_{\varepsilon}\right) D u_{\varepsilon} D u_{\varepsilon}}{\left(\varepsilon+u_{\varepsilon}\right)^{\theta}} \mathrm{e}^{-b \frac{H_{\varepsilon}\left(u_{\varepsilon}\right)}{\alpha}} \phi-\int_{\Omega} \frac{Q\left(x, u_{\varepsilon}\right) D u_{\varepsilon} D u_{\varepsilon}}{\left(\varepsilon+u_{\varepsilon}\right)^{\theta}} \mathrm{e}^{-b \frac{H_{\varepsilon}\left(u_{\varepsilon}\right)}{\alpha}} \phi \geq 0 .
\end{aligned}
$$

Then it follows

$$
\int_{\Omega} M\left(x, u_{\varepsilon}\right) D u_{\varepsilon} D \phi \mathrm{e}^{-b \frac{H_{\varepsilon}\left(u_{\varepsilon}\right)}{\alpha}} \geq \int_{\Omega} f_{\varepsilon} \mathrm{e}^{-b \frac{H_{\varepsilon}\left(u_{\varepsilon}\right)}{\alpha} \phi} .
$$

We can pass to the limit, since $M\left(x, u_{\varepsilon}\right) D u_{\varepsilon}$ converges weakly in $L^{2}$ to $M(x, u) D u$ (recall that the matrix $M$ is bounded). Therefore

$$
\int_{\Omega} M(x, u) D u D \phi \mathrm{e}^{-b \frac{H_{0}(u)}{\alpha}} \geq \int_{\Omega} \frac{T_{1}[f]}{\mathrm{e}^{\frac{b H_{0}(u)}{\alpha}}} \phi, \quad \forall \phi \in \mathcal{D}(\Omega), \phi \geq 0 .
$$

Define $P(s)=\int_{0}^{s} \mathrm{e}^{-b \frac{H_{0}(t)}{\alpha}} \mathrm{d} t$ and $w(x)=P(u(x))=\int_{0}^{u(x)} \mathrm{e}^{-b \frac{H_{0}(t)}{\alpha}} \mathrm{d} t$. The comparison principle in $W_{0}^{1,2}(\Omega)$ says that $w(x) \geq z(x)$, where $z$ is the bounded weak solution of

$$
z \in W_{0}^{1,2}(\Omega):-\operatorname{div}(M(x, u(x)) D z)=\frac{T_{1}[f]}{\mathrm{e}^{b \frac{H_{0}(u)}{\alpha}}} .
$$

The strong maximum principle for weak solutions implies $z>0$ in $\Omega$ (see [31,32]) and so $P(u)>0$ and also $u>0$ in $\Omega$, since the real function $P(s)$ is strictly increasing. Thus we have no problems to pass to the limit. 
Now use $\mathrm{e}^{\frac{-b H_{\varepsilon}\left(u_{\varepsilon}\right)}{\alpha}} \mathrm{e}^{\frac{b H_{\varepsilon}\left(T_{j}(u)\right)}{\alpha}} \phi, \quad \phi \geq 0, \quad \phi \in W_{0}^{1,2}(\Omega) \cap L^{\infty}(\Omega)$, as test function. Then

$$
\mid \begin{aligned}
& \frac{b}{\alpha} \int_{\Omega} M\left(x, u_{\varepsilon}\right) D u_{\varepsilon} D T_{j}(u) H_{\varepsilon}^{\prime}(u) \mathrm{e}^{-b H_{\varepsilon}\left(u_{\varepsilon}\right)} \mathrm{e}^{\frac{b H_{\varepsilon}\left(T_{j}(u)\right)}{\alpha}} \phi \\
& +\int_{\Omega} M\left(x, u_{\varepsilon}\right) D u_{\varepsilon} D \phi \mathrm{e}^{-b H_{\varepsilon}\left(u_{\varepsilon}\right)} \mathrm{e}^{\frac{b H_{\varepsilon}\left(T_{j}(u)\right)}{\alpha}} \\
& =\int_{\Omega} f_{\varepsilon} \mathrm{e}^{-b H_{\varepsilon}\left(u_{\varepsilon}\right)} \mathrm{e}^{\frac{b H_{\varepsilon}\left(T_{j}(u)\right)}{\alpha}} \phi \\
& +\int_{\Omega}\left\{\frac{b}{\alpha} M\left(x, u_{\varepsilon}\right) D u_{\varepsilon} D u_{\varepsilon} H_{\varepsilon}^{\prime}\left(u_{\varepsilon}\right) \mathrm{e}^{-b H_{\varepsilon}\left(u_{\varepsilon}\right)} \mathrm{e}^{\frac{b H_{\varepsilon}\left(T_{j}(u)\right)}{\alpha}} \phi\right. \\
& \left.\quad-\frac{Q\left(x, u_{\varepsilon}\right) D u_{\varepsilon} D u_{\varepsilon}}{\left(\varepsilon+u_{\varepsilon}\right)^{\theta}} \mathrm{e}^{-b H_{\varepsilon}\left(u_{\varepsilon}\right)} \mathrm{e}^{\frac{b H_{\varepsilon}\left(T_{j}(u)\right)}{\alpha}} \phi\right\} .
\end{aligned}
$$

Note that the last integrand is positive: we can use Fatou Lemma in the right hand side (thanks to the fact that $u>0$ in $\Omega$ ); we can handle the left hand side as in the previous section, as $\varepsilon \rightarrow 0$ and then as $j \rightarrow \infty$. Since $\lim _{\varepsilon \rightarrow 0} \mathrm{e}^{\frac{-b H_{\varepsilon}\left(u_{\varepsilon}\right)}{\alpha}} \mathrm{e}^{\frac{b H_{\varepsilon}\left(T_{j}(u)\right)}{\alpha}}=\mathrm{e}^{\frac{-b H_{0}(u)}{\alpha}} \mathrm{e}^{\frac{b H_{0}\left(T_{j}(u)\right)}{\alpha}} \leq 1$, and $\lim _{j \rightarrow \infty} \mathrm{e}^{-b H_{0}(u)} \mathrm{e}^{b H_{0}\left(T_{j}(u)\right)}=1$ we have

$$
\mid \begin{aligned}
& \int_{\Omega} M(x, u) D u D u \frac{b}{\alpha u^{\theta}} \phi+\int_{\Omega} M(x, u) D u D \phi \\
& \geq \int_{\Omega} f \phi+\int_{\Omega}\left\{\frac{b}{\alpha u^{\theta}} M(x, u) D u D u \phi-\frac{Q(x, u) D u D u}{u^{\theta}} \phi\right\} .
\end{aligned}
$$

Then we get

$$
\int_{\Omega} M(x, u) D u D \phi \geq \int_{\Omega} f \phi-\frac{Q(x, u) D u D u}{u^{\theta}} \phi .
$$

On the other hand the opposite inequality follows from

$$
\int_{\Omega} M\left(x, u_{\varepsilon}\right) D u_{\varepsilon} D \phi+\int_{\Omega} \frac{Q\left(x, u_{\varepsilon}\right) D u_{\varepsilon} D u_{\varepsilon}}{\left(\varepsilon+u_{\varepsilon}\right)^{\theta}} \phi=\int_{\Omega} f_{\varepsilon} \phi
$$

$\left(0 \leq \phi \in W_{0}^{1,2}(\Omega) \cap L^{\infty}(\Omega)\right)$ thanks to Fatou Lemma and the weak $L^{2}$ convergence of $M\left(x, u_{\varepsilon}\right) D u_{\varepsilon}$.

\section{LiNEAR GROWTH SINGULARITY}

Theorem 4.1. Let

$$
0 \leq f \in L^{m}(\Omega), \quad m \geq \frac{2 N}{N+2}, \quad f \not \equiv 0 .
$$

Under the assumption (1.5), with $\beta(s) \leq \beta_{0} \in \mathbb{R}^{+},(3.3), \alpha>2 b$ and (4.1), there exists $u \in W_{0}^{1,2}(\Omega), u>0$ in $\Omega$, with $\frac{Q(x, u) D u D u}{u} \in L^{1}(\Omega)$, weak solution of the singular-quadratic Dirichlet problem

$$
\int_{\Omega} M(x, u) D u D \phi+\int_{\Omega} \frac{Q(x, u) D u D u}{u} \phi=\int_{\Omega} f \phi, \quad \forall \phi \in W_{0}^{1,2}(\Omega) \cap L^{\infty}(\Omega) .
$$


Proof. Define $u_{\varepsilon}$ :

$$
u_{\varepsilon} \in W_{0}^{1,2}(\Omega):-\operatorname{div}\left(M\left(x, u_{\varepsilon}\right) D u_{\varepsilon}\right)+\frac{Q\left(x, u_{\varepsilon}\right) D u_{\varepsilon} D u_{\varepsilon}}{\left(\varepsilon+u_{\varepsilon}\right)}=f_{\varepsilon} .
$$

Again $\left\{f_{\varepsilon}\right\}$ is a sequence of bounded functions converging to $f$ in $L^{m}(\Omega), 0 \leq f_{\varepsilon} \leq f ;$ e.g. $f_{\varepsilon}=T_{\frac{1}{\varepsilon}}(f)$. As in the previous sections, $u_{\varepsilon} \in L^{\infty}(\Omega)$ and it is possible to prove that the sequence $\left\{u_{\varepsilon}\right\}$ is bounded in $W_{0}^{1,2}(\Omega)$ (thus $u_{\varepsilon} \rightarrow u$, for some $u \in W_{0}^{1,2}(\Omega)$ ), that the sequence of the lower order terms is bounded in $L^{1}(\Omega)$,

$$
\int_{\Omega} \frac{Q\left(x, u_{\varepsilon}\right) D u_{\varepsilon} D u_{\varepsilon}}{\left(\varepsilon+u_{\varepsilon}\right)} \leq \int_{\Omega} f_{\varepsilon} \leq \int_{\Omega} f,
$$

and that $D u_{\varepsilon}(x)$ converges a.e. to $D u(x)$. Moreover (4.4) implies

$$
a \int_{\Omega} \frac{\left|D u_{\varepsilon}\right|^{2}}{\left(\varepsilon+u_{\varepsilon}\right)} \leq \int_{\Omega} f
$$

Now we shall prove that $u>0$ in $\Omega$. Let $\phi \in \mathcal{D}(\Omega), \phi \geq 0$, and use $\frac{\phi}{\left(\varepsilon+u_{\varepsilon}\right)^{\frac{b}{\alpha}}}$ as test function to obtain

$$
\mid \begin{aligned}
& \int_{\Omega}\left[M\left(x, u_{\varepsilon}\right) D u_{\varepsilon} D \phi\right] \frac{1}{\left(\varepsilon+u_{\varepsilon}\right)^{\frac{b}{\alpha}}}-\int_{\Omega} f_{\varepsilon} \frac{1}{\left(\varepsilon+u_{\varepsilon}\right)^{\frac{b}{\alpha}}} \phi \\
& =\int_{\Omega} \frac{b}{\alpha} M\left(x, u_{\varepsilon}\right) D u_{\varepsilon} D u_{\varepsilon} \frac{1}{\left(\varepsilon+u_{\varepsilon}\right)^{\frac{b}{\alpha}+1}} \phi \\
& -\int_{\Omega} \frac{Q\left(x, u_{\varepsilon}\right) D u_{\varepsilon} D u_{\varepsilon}}{\left(\varepsilon+u_{\varepsilon}\right)^{\frac{b}{\alpha}+1}} \phi \geq 0 .
\end{aligned}
$$

Let $L>0$ be such that the measure of the set $\{x \in \Omega: u(x)=L\}$ is zero; all except countably many $L$ are such that this holds, since $u \in L^{1}(\Omega)$. Since $u_{\varepsilon}(x) \rightarrow u(x)$ a.e., thanks to the choice of $L, \chi_{\left\{u_{\varepsilon} \leq L\right\}}(x) \rightarrow \chi_{\{u \leq L\}}(x)$ a.e. in $\Omega$ and so

$$
\int_{\Omega}\left[M\left(x, u_{\varepsilon}\right) D u_{\varepsilon} D \phi\right] \frac{1}{\left(\varepsilon+u_{\varepsilon}\right)^{\frac{b}{\alpha}}} \geq \int_{\Omega} \chi_{\left\{u_{\varepsilon} \leq L\right\}} f_{1}(x) \frac{1}{(1+L)^{\frac{b}{\alpha}}} \phi .
$$

Here we use the the fact that the assumption $\alpha>2 b$ implies that $1-\frac{b}{\alpha}>0$ and we obtain

$$
\int_{\Omega} M\left(x, u_{\varepsilon}\right) D\left[\left(\varepsilon+u_{\varepsilon}\right)^{1-\frac{b}{\alpha}}-\varepsilon^{1-\frac{b}{\alpha}}\right] D \phi \geq \int_{\Omega} \chi_{\left\{u_{\varepsilon} \leq L\right\}} f_{1}(x) \frac{1-\frac{b}{\alpha}}{(1+L)^{\frac{b}{\alpha}}} \phi .
$$

The comparison principle in $W_{0}^{1,2}(\Omega)$ says that $\left[\left(\varepsilon+u_{\varepsilon}\right)^{1-\frac{b}{\alpha}}-\varepsilon^{1-\frac{b}{\alpha}}\right] \geq z_{\varepsilon}$, where $z_{\varepsilon}$ is the weak solution of

$$
z_{\varepsilon} \in W_{0}^{1,2}(\Omega):-\operatorname{div}\left(M\left(x, u_{\varepsilon}\right) D z_{\varepsilon}\right)=\chi_{\left\{u_{\varepsilon} \leq L\right\}} f_{1}(x) \frac{1-\frac{b}{\alpha}}{(1+L)^{\frac{b}{\alpha}}} .
$$

It is easy to see that $z_{\varepsilon}$ converges strongly in $W_{0}^{1,2}(\Omega)$ to $z_{0}$, the solution of

$$
z_{0} \in W_{0}^{1,2}(\Omega):-\operatorname{div}\left(M(x, u) D z_{0}\right)=\chi_{\{u \leq L\}} f_{1}(x) \frac{1-\frac{b}{\alpha}}{(1+L)^{\frac{b}{\alpha}}} .
$$


The strong maximum principle for weak solutions implies $z_{0}>0$ in $\Omega$ (see [31,32]). If we pass to the limit in the inequality

$$
0<z_{\varepsilon}(x) \leq\left[\left(\varepsilon+u_{\varepsilon}(x)\right)^{1-\frac{b}{\alpha}}-\varepsilon^{1-\frac{b}{\alpha}}\right],
$$

the almost everywhere convergence of $u_{\varepsilon}(x)$ to $u(x)$ then guarantees that $0<z_{0}(x) \leq u(x)^{1-\frac{b}{\alpha}}$. Thus also

$$
u>0 \text { in } \Omega
$$

since $1-\frac{b}{\alpha}>0$, and we have no problems to pass to the limit. From (4.4) and (4.5) we have

$$
a \int_{\Omega} \frac{|D u|^{2}}{u} \int_{\Omega} \frac{Q(x, u) D u D u}{u} \leq \int_{\Omega} f
$$

and that

$$
\frac{D u_{\varepsilon}}{\sqrt{\varepsilon+u_{\varepsilon}}} \rightarrow \frac{D u}{\sqrt{u}} \text { converges weakly in } L^{2} .
$$

As in the previous section, we now pass to the limit in (4.3). We begin with the first half of the result. Use as test function $0 \leq \phi \in W_{0}^{1,2}(\Omega) \cap L^{\infty}(\Omega)$ to obtain

$$
\int_{\Omega} M\left(x, u_{\varepsilon}\right) D u_{\varepsilon} D \phi+\int_{\Omega} \frac{Q\left(x, u_{\varepsilon}\right) D u_{\varepsilon} D u_{\varepsilon}}{\left(\varepsilon+u_{\varepsilon}\right)} \phi=\int_{\Omega} f \phi
$$

and Fatou Lemma as $\varepsilon \rightarrow 0$. Then we deduce

$$
\int_{\Omega} M(x, u) D u D \phi+\int_{\Omega} \frac{Q(x, u) D u D u}{u} \phi \leq \int_{\Omega} f \phi .
$$

Now we use $v_{\varepsilon}=\frac{(\varepsilon+u)^{\frac{1}{2}}}{\left(\varepsilon+u_{\varepsilon}\right)^{\frac{1}{2}}} \phi$ as test function in (4.3).

$$
\mid \begin{aligned}
& \frac{1}{2} \int_{\Omega} M\left(x, u_{\varepsilon}\right) \frac{D u_{\varepsilon}}{\left(\varepsilon+u_{\varepsilon}\right)^{\frac{1}{2}}} \frac{D u}{(\varepsilon+u)^{\frac{1}{2}}} \phi \\
& +\int_{\Omega} M\left(x, u_{\varepsilon}\right) \frac{D u_{\varepsilon}}{\left(\varepsilon+u_{\varepsilon}\right)^{\frac{1}{2}}} D \phi(\varepsilon+u)^{\frac{1}{2}}=\int_{\Omega} f_{\varepsilon} \frac{(\varepsilon+u)^{\frac{1}{2}}}{\left(\varepsilon+u_{\varepsilon}\right)^{\frac{1}{2}}} \phi \\
& +\int_{\Omega}\left[\frac{1}{2} M\left(x, u_{\varepsilon}\right) D u_{\varepsilon} D u_{\varepsilon}-Q\left(x, u_{\varepsilon}\right) D u_{\varepsilon} D u_{\varepsilon}\right] \frac{(\varepsilon+u)^{\frac{1}{2}}}{\left(\varepsilon+u_{\varepsilon}\right)^{\frac{1}{2}+1}} \phi .
\end{aligned}
$$

Note that the last integrand is positive thanks to the assumption $\alpha \geq 2 b$. Fatou Lemma once again, as well as (4.10) imply, as $\varepsilon \rightarrow 0$,

$$
\mid \begin{aligned}
& \frac{b}{\alpha} \int_{\Omega} M(x, u) D u \frac{D u}{u} \phi+\int_{\Omega} M(x, u) D u D \phi \\
& \geq \int_{\Omega} f \phi+\int_{\Omega}\left[\frac{1}{2} M(x, u) D u D u-Q(x, u) D u D u\right] \frac{1}{u} \phi,
\end{aligned}
$$

that is the second half of the result:

$$
\int_{\Omega} M(x, u) D u D \phi \geq \int_{\Omega} f \phi-\int_{\Omega} \frac{Q(x, u) D u D u}{u} \phi .
$$


Remark 4.1. Note that the assumption $f(x) \geq 0$ allows to have a right hand side zero on a set of positive measure.

Remark 4.2. In this section, we assume $\theta=1$. Note that assumption (4.1) is exactly assumption (3.1) with $\theta=1$.

Remark 4.3. Note that in order to prove (4.8) only inequality $\alpha \geq b$ is needed, instead of $\alpha \geq 2 b$, which is the assumption of the previous theorem.

\section{REMARKS ON TWO RELATED PROBLEMS}

\subsection{Remarks on a related semilinear problem}

Consider here the boundary value problem (4.2) in the simple case

$$
u>0 \text { in } \Omega:\left\{\begin{array}{cl}
-\alpha \Delta u+\frac{|D u|^{2}}{u}=f(x) & \text { in } \Omega, \\
u=0 & \text { on } \partial \Omega,
\end{array}\right.
$$

where $b=1$ and $\alpha \geq 2$ and $0 \leq f \in L^{\frac{2 N}{N+2}}(\Omega)$, as in Theorem 4.1.

We look for the equation satisfied by $z(x)=q(u(x))$, where $q(s)$ is a real smooth function. We have $-\frac{\alpha \Delta z}{q^{\prime}(u)}=-\alpha \frac{q^{\prime \prime}(u)|D u|^{2}}{q^{\prime}(u)}-\alpha \Delta u$. Choose $q(t)$ such that $-\alpha \frac{q^{\prime \prime}(t)}{q^{\prime}(t)}=\frac{1}{t}$, so that $-\alpha \Delta z=f(x) q^{\prime}\left(q^{-1}(z)\right)$ and $\log q^{\prime}(t)=\log \left[\frac{1}{t^{1 / \alpha}}\right]: q^{\prime}(t)=\frac{1}{t^{1 / \alpha}}$. Then the new problem, which depends on $\alpha$, at least formally is

$$
-\Delta z=f(x) \frac{1}{\left(q^{-1}(z)\right)^{\frac{1}{\alpha}}}
$$

since, for $\alpha>1, q(t)=\frac{\alpha}{\alpha-1} t^{1-1 / \alpha}, q^{-1}(t)=\left[\left(\frac{\alpha-1}{\alpha}\right) t\right]^{\frac{\alpha}{\alpha-1}}$ and $z=\frac{\alpha}{\alpha-1} u^{1-1 / \alpha}$ is such that

$$
\left\{\begin{array}{l}
z>0 \text { in } \Omega: \quad-\Delta z=\frac{C_{\alpha} f(x)}{z^{\frac{1}{\alpha-1}}}, \quad \text { in } \Omega \\
z=0, \quad \text { on } \partial \Omega .
\end{array}\right.
$$

The previous boundary value problem can be seen as the Euler-Lagrange equation of the coercive functional

$$
\frac{1}{2} \int_{\Omega}|D v|^{2}-\tilde{C}_{\alpha} \int_{\Omega} f(x) v^{\frac{\alpha-2}{\alpha-1}}, \quad \tilde{C}_{\alpha}>0, f(x) \geq 0, \alpha \geq 2 .
$$

Note that the problem (5.1) has been extensively studied at least with $f$ bounded (see e.g. the pioneering paper [18] and also [24]). Moreover, in the spirit of this section, it is important to recall [28].

Moreover, if $\alpha \geq 2$, we are able to say that $z=\frac{\alpha}{\alpha-1} u^{1-1 / \alpha}$, solution of (5.1), belongs to $W_{0}^{1,2}(\Omega)$, as consequence of (4.9).

On the other hand with the choice of $z$ as test function in (5.1), we have

$$
\int_{\Omega}|D z|^{2}=C_{\alpha} \int_{\Omega} f(x) z^{\frac{\alpha-2}{\alpha-1}}
$$


and, always if $\alpha \geq 2$,

$$
C_{1}\left[\int_{\Omega} z^{2^{*}}\right]^{\frac{N-2}{N}} \leq\left[\int_{\Omega} z^{2^{*}}\right]^{\frac{(N-2)(\alpha-2)}{2 N(\alpha-1)}}\left[\int_{\Omega} f(x)^{\frac{2 N(\alpha-1)}{N \alpha+2 \alpha-4}}\right]^{\frac{N \alpha+2 \alpha-4}{2 N(\alpha-1)}} .
$$

Note that $\frac{2 N(\alpha-1)}{N \alpha+2 \alpha-4} \leq \frac{2 N}{N+2}$ and $\frac{(N-2)(\alpha-2)}{2 N(\alpha-1)}<\frac{N-2}{N}$.

So that

$$
\|z\|_{2^{*}} \leq C_{0}\|f\|_{m} .
$$

Thus we have an a priori estimate on $z$, solution of (5.1), in the Sobolev space $W_{0}^{1,2}(\Omega)$, if $\alpha \geq 2$ and $m \geq \frac{2 N}{N+2}$.

If $\alpha=1$, the new problem is

$$
\left\{\begin{array}{l}
-\Delta z=\frac{f(x)}{\mathrm{e}^{z}}, \quad \text { in } \Omega, \\
z=-\infty,
\end{array} \text { on } \partial \Omega,\right.
$$

while if $0<\alpha<1$

$$
\begin{cases}z<0 \text { in } \Omega: & -\Delta z=f(x) \tilde{C}_{\alpha}|z|^{\frac{1}{1-\alpha}}, \quad \text { in } \Omega, \\ z=-\infty, & \text { on } \partial \Omega,\end{cases}
$$

where $\tilde{C}_{\alpha}>0$.

In both above problems we point out the explosive boundary condition on $z$. Setting $w=-z$ both enter the framework

$$
\left\{\begin{array}{l}
-\Delta w+h(x) g(w)=0, \\
w \rightarrow+\infty, \quad \text { on } \partial \Omega,
\end{array}\right.
$$

where $g(s)$ satisfies the so-called Keller-Osserman condition.

Such problems have a huge literature since the first basic study in $[23,26]$ to the recent results of [28].

\subsection{Remarks on a related porous media problem}

Consider here the boundary value problem (4.2) in the simple case

$$
u>0 \text { in } \Omega:\left\{\begin{array}{cl}
-\operatorname{div}(M(x) D u)+\frac{|D u|^{2}}{u}=2 f(x) & \text { in } \Omega, \\
u=0 & \text { on } \partial \Omega,
\end{array}\right.
$$

where $b=1$ and $\alpha \geq 2$ and $0 \leq f \in L^{\frac{2 N}{N+2}}(\Omega)$. Thanks to Theorem 4.1, we can prove the existence of a solution $u \in W_{0}^{1,2}(\Omega)$, such that $\frac{|D u|^{2}}{u} \in L^{1}(\Omega)$. Define now $z=\sqrt{u}$. Then the function $z$ is solution of

$$
0<z \in W_{0}^{1,2}(\Omega):\left\{\begin{array}{cl}
-\operatorname{div}(M(x) z D z)+2|D z|^{2}=f(x) & \text { in } \Omega, \\
z=0 & \text { on } \partial \Omega,
\end{array}\right.
$$

with $\alpha \geq 2$ and $0 \leq f \in L^{\frac{2 N}{N+2}}(\Omega)$. Such kind of problems has been studied in [33]. 
Acknowledgements. The results of this paper were presented in a lecture at the International Workshop ANCPDE'07, dedicated to Jean-Pierre Puel for his 60th birthday (Pont-à-Mousson; June 25, 2007).

I wish to thank David Arcoya (who introduced me to the subject of Sect. 4), Daniela Giachetti (for informations about a related problem studied in [22]), Luigi Orsina, Alessio Porretta, Michaela Porzio (for some very useful discussions about the present paper), Hotel Ipanema Inn (for Th. 3.1 was successfully proved there) and Hotel Leblon Inn.

\section{REFERENCES}

[1] D. Arcoya, S. Barile and P.J. Martinez-Aparicio, Singular quasilinear equations with quadratic growth in the gradient without sign condition. Preprint.

[2] D. Arcoya and P.J. Martinez-Aparicio, Quasilinear equations with natural growth Rev. Mat. Iberoamericana (to appear).

[3] D. Arcoya, J. Carmona, T. Leonori, P.J. Martínez, L. Orsina and F. Petitta, Quadratic quasilinear equations with general singularities. Preprint.

[4] A. Bensoussan, L. Boccardo and F. Murat, On a nonlinear partial differential equation having natural growth terms and unbounded solution. Ann. Inst. H. Poincaré Anal. Non Linéaire 5 (1988) 347-364.

[5] L. Boccardo, Some nonlinear Dirichlet problems in $L^{1}$ involving lower order terms in divergence form, in Progress in elliptic and parabolic partial differential equations (Capri, 1994), Pitman Res. Notes Math. Ser. 350, Longman, Harlow (1996) 43-57.

[6] L. Boccardo, Positive solutions for some quasilinear elliptic equations with natural growths. Atti Accad. Naz. Lincei 11 (2000) 31-39.

[7] L. Boccardo, Hardy potential and quasi-linear elliptic problems having natural growth terms, in Proceedings of the Conference held in Gaeta on the occasion of the 60th birthday of Haim Brezis, Progr. Nonlinear Differential Equations Appl. 63, Birkhauser, Basel (2005) 67-82.

[8] L. Boccardo and T. Gallouët, Nonlinear elliptic and parabolic equations involving measure data. J. Funct. Anal. 87 (1989) 149-169.

[9] L. Boccardo and T. Gallouët, Strongly nonlinear elliptic equations having natural growth terms and $L^{1}$ data. Nonlinear Anal. 19 (1992) 573-579.

[10] L. Boccardo, T. Gallouët and L. Orsina, Existence and nonexistence of solutions for some nonlinear elliptic equations. J. Anal. Math. 73 (1997) 203-223.

[11] L. Boccardo and D. Giachetti, Existence results via regularity for some nonlinear elliptic problems. Comm. Partial Diff. Eq. 14 (1989) 663-680.

[12] L. Boccardo and F. Murat, Almost everywhere convergence of the gradients of solutions to elliptic and parabolic equations. Nonlinear Anal. TMA 19 (1992) 581-597.

[13] L. Boccardo, F. Murat and J.-P. Puel, Existence de solutions non bornées pour certaines équations quasi-linéaires. Portugaliae Math. 41 (1982) 507-534.

[14] L. Boccardo, F. Murat and J.-P. Puel, Résultats d'existence pour certains problèmes elliptiques quasi linéaires. Ann. Sc. Norm. Sup. Pisa 11 (1984) 213-235.

[15] L. Boccardo, F. Murat and J.-P. Puel, Existence of bounded solutions for nonlinear elliptic unilateral problems. Ann. Mat. Pura Appl. 152 (1988) 183-196.

[16] L. Boccardo, F. Murat and J.-P. Puel, $L^{\infty}$-estimates for some nonlinear partial differential equations and application to an existence result. SIAM J. Math. Anal. 23 (1992) 326-333.

[17] H. Brezis and L. Nirenberg, Removable singularities for nonlinear elliptic equations. Topol. Methods Nonlinear Anal. 9 (1997) 201-219.

[18] M.G. Crandall, P.H. Rabinowitz and L. Tartar, On a Dirichlet problem with a singular nonlinearity. Comm. Partial Diff. Eq. 2 (1977) 193-222.

[19] A. Dall'Aglio, D. Giachetti and J.-P. Puel, Nonlinear elliptic equations with natural growth in general domains. Ann. Mat. Pura Appl. 181 (2002) 407-426.

[20] A. Dall'Aglio, V. De Cicco, D. Giachetti and J.-P. Puel, Existence of bounded solutions for nonlinear elliptic equations in unbounded domains. NoDEA 11 (2004) 431-450.

[21] T. Del Vecchio, Strongly nonlinear problems with Hamiltonian having natural growth. Houston J. Math. 16 (1990) 7-24.

[22] D. Giachetti and F. Murat, Personal communication.

[23] J.B. Keller, On solutions of $\Delta u=f(u)$. Commun. Pure Appl. Math. 10 (1957) 503-510.

[24] A.C. Lazer and P.J. McKenna, On a singular nonlinear elliptic boundary-value problem. Proc. Amer. Math. Soc. 111 (1991) $721-730$.

[25] T. Leonori, Large solutions for a class of nonlinear elliptic equations with gradient terms. Adv. Nonlinear Stud. 7 (2007) 237-269.

[26] R. Osserman, On the inequality $\Delta u \geq f(u)$. Pacific J. Math. 7 (1957) 1641-1647.

[27] A. Porretta, Existence for elliptic equations in $L^{1}$ having lower order terms with natural growth. Portugaliae Math. 57 (2000) $179-190$ 
[28] A. Porretta, A local estimates and large solutions for some elliptic equations with absorption. Adv. Differential Equations 9 (2004) 329-351.

[29] A. Porretta and S. Segura de Leon, Nonlinear elliptic equations having a gradient term with natural growth. J. Math. Pures Appl. 85 (2006) 465-492.

[30] J.-P. Puel, Existence, comportement à l'infini et stabilité dans certains problèmes quasilinéaires elliptiques et paraboliques d'ordre 2. Ann. Scuola Norm. Sup. Pisa Cl. Sci. (4) 3 (1976) 89-119.

[31] G. Stampacchia, Le problème de Dirichlet pour les équations elliptiques du second ordre à coefficients discontinus. Ann. Inst. Fourier (Grenoble) 15 (1965) 189-258.

[32] N.S. Trudinger, On Harnack type inequalities and their application to quasilinear elliptic equations. Comm. Pure Appl. Math. 20 (1967) $721-747$.

[33] J.L. Vazquez, The Porous Medium Equation: Mathematical Theory, Oxford Mathematical Monographs. Oxford University Press, Oxford (2007). 